\title{
Arm Type Code
}

National Cancer Institute

\section{Source}

National Cancer Institute. Arm Type Code. NCI Thesaurus. Code C93732.

A coded value specifying the kind of arm. 